\title{
H1N1 - wo stehen wir im Moment?
}

\author{
Tobias Welte
}

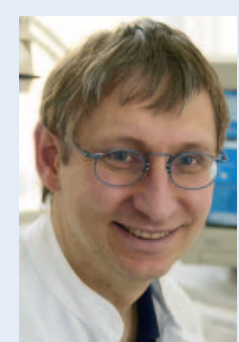

Tobias Welte

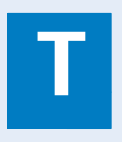

rotz Bundestagswahl, Finanzkrise und Afghanistan-Konflikt wurde kein anderes Thema zumindest bis kurz vor Weihnachten - so intensiv und kontrovers in den Medien behandelt wie die Infektion mit H1N1. Dabei ist es bedauerlich, wer sich - bar jeden Wissens über die Art des Virus, seine Gefahren und die Möglichkeiten von Therapie und Prävention - genötigt sah, Kommentare zu dieser Erkrankung öffentlich abzugeben. Dabei ist man ja durchaus von Politikern gewohnt, dass geredet wird ohne Nachzudenken. Besonders zu kritisieren sind diejenigen unserer ärztlichen Kollegen, die jede Gelegenheit genutzt haben, in teilweise unverantwortlicher Art und Weise, Ihre persönliche - meist auf unzureichender Information beruhende - Meinung zum Besten zu geben und damit das öffentliche Verhalten wesentlich beeinflusst haben. Dass sich zudem selbst sogenannte seriösen Zeitungen wie die FAZ oder die Süddeutsche Zeitung mehr für Tote interessieren und Endzeitszenarien herbeireden, statt - manchmal unspektakuläre Wahrheiten - zu berichten, ist beängstigend, weil der Einfluss der Medien auf das Verhalten der Bevölkerung so groß ist.

Inzwischen ist - ähnlich wie es schon vor Jahren bei BSE und SARS der Fall war (wer weiß eigentlich noch wirklich, worum es bei diesen Erkrankungen ging?) H1N1 aus den Medien verschwunden, es wird nur noch darüber diskutiert, wie man die Kosten der voreilig angelegten Impfstoff- und Neuraminidaselager reduziert. Ist das Problem der H1N1-Pandemie damit wirklich gelöst und müssen wir uns keine weiteren Gedanken für die Zukunft machen? Gerade dies scheint nicht der Fall und viele am Beispiel H1N1 zutage getretenen Schwächen unseres Gesundheitssystems müssen jetzt intensiv diskutiert werden, auch im Bereich der Intensivmedizin und Notfallmedizin.

Die H1N1-Pandemie ist bisher wesentlich milder verlaufen als von vielen Experten prognostiziert und befürchtet worden war. Hierfür dürfte es verschiedene Gründe geben, wobei 3 Dinge wesentlich sind. Der Vi- rus war primär weniger pathogen als befürchtet, eine Zunahme der Pathogenität durch eine Veränderung des Virus ist bisher nicht zu beobachten. Die Kontagiosität von H1N1 war geringer als angenommen. Wirklich entscheidend dürfte allerdings gewesen sein, dass kaum ältere Menschen über 65 Jahre erkrankten, weil möglicherweise eine in den 50er-Jahren dem jetzigen H1N1 ähnliche Virusvariante für eine Immunität bei Älteren gesorgt hat.

Da auf epidemiologisch aussagekräftige Screening-Untersuchungen aus Kostengründen frühzeitig verzichtet wurde, weiß niemand, wie hoch die Inzidenz an H1N1Fällen wirklich ist. Insgesamt muss man jedoch weltweit mit vielen Millionen Erkrankten rechnen, von denen jedoch nur ein sehr geringer Prozentsatz wirklich ernsthaft erkrankt ist. Durch die Entwicklung auf der Südhalbkugel, auf der der Winter vorbei ist, die Zahl der Erkrankungen fast auf Null zurückgegangen ist und damit ein guter Überblick vorliegt, können wir in etwa abschätzen, mit welcher weiteren Entwicklung in Europa gerechnet werden muss.

Die besten Zahlen liegen zurzeit für Australien und Neuseeland vor, weil hier im Rahmen eines Netzwerks aller Intensivstationen dieser Länder, die schweren Fälle vollständig erfasst und ausgewertet wurden. Danach wurden 722 Patienten intensivmedizinisch behandelt, was einer Rate von 28,7 Fällen pro 1 Million Einwohner entspricht. Bei einer mittleren Intensivaufenthaltsdauer dieser Patienten von 7 Tagen bedeutete dies, dass pro Tag maximal 7,4 Intensivbetten pro 1 Million Einwohner mit H1N1-Patienten belegt waren.

Etwa zwei Drittel aller Intensivpatienten musste beatmet werden, die Sterblichkeit war mit 14,3\% überraschend gering, wobei sich jedoch bei Publikation der Daten noch mehr als 15\% der Patienten im Krankenhaus befanden.

Das Risikofaktorenprofil der intensivmedizinisch betreuten Patienten weicht überraschend deutlich von 
üblichen Risikoprofilen für Infektionskrankheiten ab. Schwangere (9,1\% der Intensivpatienten) waren genauso wie stark übergewichtige Patienten $(\mathrm{BMI}>35)$ überproportional häufig von einem schweren Krankheitsverlauf betroffen, ohne dass die Gründe dafür wirklich verstanden wären. Von den sonst bekannten Risikofaktoren für die Entwicklung einer schweren Pneumonie spielten nur chronische Atemwegserkrankungen, wie Asthma und COPD, und Diabetes mellitus eine wesentliche Rolle. Die Mehrzahl der intensivpflichtigen Patienten war jedoch jung und ohne bekannte Vorerkrankungen. Diese Patienten zeigten innerhalb weniger Tage aus voller Gesundheit heraus ein ARDS-ähnliches Bild mit respiratorischem Versagen, ein Multiorganversagen war eher selten zu beobachten.

Gerade für diese jungen, primär gesunden Patienten wurden alle zur Verfügung stehenden Therapiemöglichkeiten ausgeschöpft, insbesondere kam der extrakorporalen Membranoxigenierung (ECMO) zur Verbesserung der Oxigenierung eine wesentliche Bedeutung zu. Nie zuvor konnte in so kurzer Zeit von Erfahrungen mit ECMO an einem derart homogenen Patientenkollektiv berichtet werden.

Die im JAMA publizierten Erfahrungen der Australier und Neuseeländer - insgesamt 68 Patienten mit schwerem Influenza-assoziiertem ARDS, die mit ECMO behandelt wurden - belegten dabei, dass gerade bei Patienten mit akuten, perspektivisch voll reversiblen Erkrankungsbildern, bei denen unter Ausschöpfung aller anerkannten Beatmungsmöglichkeiten keine ausreichende Oxigenierung zu erreichen ist, ECMO zum Überleben von Patienten beitragen kann. Noch nie konnte mit diesem Verfahren eine so geringe Sterblichkeit (21\%) gezeigt werden. Grund dafür dürfte einerseits sein, dass die Patienten jung und ohne KoMorbiditäten waren und damit per se eine bessere Prognose aufwiesen. Andererseits zeigt sich jedoch auch, dass die Zentralisierung eines solchen hochkomplexen Verfahrens in der Hand weniger spezialisierter Zentren, die nach standardisierten Vorgaben therapieren, zu guten Therapieerfolgen beiträgt.

In Deutschland sind Mitte November die ersten ARDSFälle aufgrund von H1N1 berichtet worden. Auch wenn die Zahl der schwer Erkrankten nur außerordentlich klein ist (wahrscheinlich unter 0,5\% aller Erkrankten), so ist sie doch aufgrund der hohen Zahl an Erkrankten numerisch nennenswert. Legen wir die australischen Zahlen zugrunde, so müssen wir in Deutschland mit weit über 2000 intensivpflichtigen Patienten und mit mehr als 200 ECMO-bedürftigen Patienten rechnen.
Hier stellen sich 2 wesentliche Fragen: Haben wir genügend qualifizierte Behandlungsplätze auf deutschen Intensivstationen zur Verfügung und erreichen die Patienten mit ECMO-Indikation auch wirklich diese Intensivstationen? Die erste Frage kann man wahrscheinlich bejahen. Das kürzlich gegründete ARDSNetzwerk hat eine Kapazität von mehr als 50 ECMOPlätzen im Regelbetrieb ermittelt, im Notfall können noch einmal 20 bis 30 Plätze zusätzlich in Betrieb genommen werden.

Die zweite Frage ist schwerer zu beantworten. Unsere Erfahrung in Hannover mit bisher mehr als 25 beatmeten an H1N1 erkrankten Patienten, davon mehr als die Hälfte mit ECMO versorgt, zeigt, dass viele Patienten erst spät - nach mehr als einer Woche Beatmung - verlegt wurden, teilweise mit hohen in- und exspiratorischen Beatmungsdrücken. ECMO ist jedoch nicht nur eine therapeutisch, sondern auch eine präventive Maßnahme, die der Lunge die Chance zur Heilung geben soll, ohne dass die Therapie/Beatmung schon irreversible Schäden gesetzt hat. Bei richtiger Indikationsstellung ist sie umso wirkungsvoller, je früher sie genutzt wird.

Mit der Gründung des ARDS-Netzwerks haben wir in Deutschland einen richtigen Weg beschritten, um die Aktivitäten zu standardisieren und zu koordinieren. Über die Webseite des Netzwerks www.ardsnetwork.de sind ECMO-Kapazitäten in Deutschland sichtbar und die Kontaktadressen abrufbar.

H1N1 wird vielleicht im Frühjahr noch einmal zurückkommen. Wenn nicht, wird es andere, neue Atemwegserreger geben, die für kritische Krankheitsverläufe verantwortlich sind. Unsere Aufgabe liegt darin begründet, ein gut funktionierendes Netzwerk mit allgemeinverbindlichen Standards für ein solch komplexes Verfahren zu etablieren, die Verteilung der Patienten zu steuern und die Sensibilität für die Möglichkeiten einer solchen Therapie auch bei mit dieser Methode nicht vertrauten Intensivmedizinern zu wecken.

In diesem Sinne bleibt viel zu tun.

\section{Korrespondenzadressen}

Prof. Dr. Tobias Welte

Klinik für Pneumologie

Medizinische Hochschule Hannover

Carl-Neuberg-Str. 1

30659 Hannover

welte.tobias@mh-hannover.de 